PROCEEDINGS OF THE

AMERICAN MATHEMATICAL SOCIETY

Volume 125, Number 10, October 1997, Pages 2855-2858

S $0002-9939(97) 03951-8$

\title{
ON THE PRODUCT PROPERTY OF THE PLURICOMPLEX GREEN FUNCTION
}

\author{
ARMEN EDIGARIAN
}

(Communicated by Eric Bedford)

Abstract. We prove that the pluricomplex Green function has the product property $g_{D_{1} \times D_{2}}=\max \left\{g_{D_{1}}, g_{D_{2}}\right\}$ for any domains $D_{1} \subset \mathbb{C}^{n}$ and $D_{2} \subset \mathbb{C}^{m}$.

Let $E$ denote the unit disc in $\mathbb{C}$. For any domain $G \subset \mathbb{C}^{n}$ define

$$
g_{D}(a, z):=\inf _{\substack{\varphi \in \mathcal{O}(E, D) \\ \varphi(0)=z \\ a \in \varphi(E)}}\left\{\prod_{\lambda \in \varphi^{-1}(a)}|\lambda|^{\operatorname{ord}_{\lambda}(\varphi-a)}\right\}, \quad a, z \in D,
$$

where $\mathcal{O}(E, D)$ denotes the set of all holomorphic mappings $E \rightarrow D$ and $\operatorname{ord}_{\lambda}(\varphi-a)$ denotes multiplicity of $\varphi-a$ at $\lambda$.

The function $g_{D}$ is proposed by Poletsky (cf. [Pol] ) and is called the pluricomplex Green function for D. We have that (see [Jar-Pfl1], Chapter IV)

$$
g_{D}(a, z)=\inf _{\substack{\varphi \in \mathcal{O}(\bar{E}, D) \\ \varphi(0)=z \\ a \in \varphi(E)}}\left\{\prod_{\lambda \in \varphi^{-1}(a)}|\lambda|^{\operatorname{ord}_{\lambda}(\varphi-a)}\right\}, \quad a, z \in D .
$$

Note that in the formula (a) we take only $\lambda \in \varphi^{-1}(a)$ such that $\lambda \in E$.

(b) For any domains $D_{1}, D_{2}$ and any holomorphic mapping $f: D_{1} \rightarrow D_{2}$ we have the following contractible property: $g_{D_{2}}(f(z), f(w)) \leq g_{D_{1}}(z, w), z, w \in D_{1}$.

The main result of the paper is the following product property.

Theorem. Let $D_{1} \subset \mathbb{C}^{n}$ and $D_{2} \subset \mathbb{C}^{m}$ be domains. Then

$$
\begin{array}{r}
g_{D_{1} \times D_{2}}\left(\left(z_{1}, w_{1}\right),\left(z_{2}, w_{2}\right)\right)=\max \left\{g_{D_{1}}\left(z_{1}, z_{2}\right), g_{D_{2}}\left(w_{1}, w_{2}\right)\right\}, \\
\quad\left(z_{1}, w_{1}\right),\left(z_{2}, w_{2}\right) \in D_{1} \times D_{2} .
\end{array}
$$

Remark. The product property for $D_{1} \times D_{2}$ for the pluricomplex Green function in the case when $D_{1}$ or $D_{2}$ is pseudoconvex was proved in [Jar-Pfl2]. Note that in [Jar-Pfl2] the authors used the description of the pluricomplex Green function given by M. Klimek.

Proof. The inequality " $\geq$ " follows from the property (b). So, we have to prove " $\leq$ ".

Received by the editors February 19, 1996.

1991 Mathematics Subject Classification. Primary 32F05, 31C10.

(C)1997 American Mathematical Society 
Let $\left(a_{1}, b_{1}\right),\left(a_{2}, b_{2}\right) \in D_{1} \times D_{2}$. If $a_{1}=a_{2}$ or $b_{1}=b_{2}$, then the required inequality follows from the property (b). So, we may assume that $a_{1} \neq a_{2}$ and $b_{1} \neq b_{2}$.

Suppose that $N \in(0,1)$ is such that

$$
\max \left\{g_{D_{1}}\left(a_{1}, a_{2}\right), g_{D_{2}}\left(b_{1}, b_{2}\right)\right\}<N .
$$

It is sufficient to prove that

$$
g_{D_{1} \times D_{2}}\left(\left(a_{1}, b_{1}\right),\left(a_{2}, b_{2}\right)\right)<N .
$$

There are holomorphic mappings $\varphi_{1}: \bar{E} \rightarrow D_{1}$ and $\varphi_{2}: \bar{E} \rightarrow D_{2}$ such that $\varphi_{1}(0)=a_{2}, \varphi_{2}(0)=b_{2}$,

$$
\prod_{\lambda \in \varphi_{1}^{-1}\left(a_{1}\right)}|\lambda|^{\operatorname{ord}_{\lambda}\left(\varphi_{1}-a_{1}\right)}<N \quad \text { and } \quad \prod_{\lambda \in \varphi_{2}^{-1}\left(b_{1}\right)}|\lambda|^{\operatorname{ord}_{\lambda}\left(\varphi_{1}-b_{1}\right)}<N .
$$

Note that $\nu:=\#\left(\varphi_{1}^{-1}\left(a_{1}\right) \cap E\right)<\infty$ and $\mu:=\#\left(\varphi_{2}^{-1}\left(b_{1}\right) \cap E\right)<\infty$. We may assume that $\varphi_{1}$ and $\varphi_{2}$ are such that $\nu$ and $\mu$ are minimal.

Let $\varphi_{1}^{-1}\left(a_{1}\right) \cap E=\left\{\zeta_{1}, \ldots, \zeta_{\nu}\right\}$ and $\varphi_{2}^{-1}\left(b_{1}\right) \cap E=\left\{\xi_{1}, \ldots, \xi_{\mu}\right\}$, where each point counts with its multiplicity. ${ }^{1}$ Since $\varphi_{1}(E) \Subset D_{1}$ and $\varphi_{2}(E) \Subset D_{2}$, we may assume that $\left|\zeta_{1}\right|<\left|\zeta_{2}\right|<\cdots<\left|\zeta_{\nu}\right|$ and $\left|\xi_{1}\right|<\left|\xi_{2}\right|<\cdots<\left|\xi_{\nu}\right|$, i.e. each point $\zeta_{j}$ and $\xi_{j}$ is with multiplicity one. ${ }^{2}$ Then

$$
\left|\zeta_{1} \ldots \zeta_{\nu}\right| \geq N\left|\zeta_{\nu}\right|^{\nu} \quad \text { and } \quad\left|\xi_{1} \ldots \xi_{\mu}\right| \geq N\left|\xi_{\mu}\right|^{\mu} .
$$

For, if $\left|\zeta_{1} \ldots \zeta_{\nu}\right|<N\left|\zeta_{\nu}\right|^{\nu}$, then we may consider the mapping $\varphi_{1}\left(\zeta_{\nu} \lambda\right)$, and it contradicts the minimality of $\nu$.

If $\left|\zeta_{1} \ldots \zeta_{\nu}\right|<\left|\xi_{1} \ldots \xi_{\mu}\right|$, then we replace $\varphi_{1}$ with the mapping $\widetilde{\varphi}_{1}(\lambda)=\varphi_{1}(t \lambda)$, where $t:=\left(\frac{\left|\zeta_{1} \ldots \zeta_{\nu}\right|}{\left|\xi_{1} \ldots \xi_{\mu}\right|}\right)^{\frac{1}{\nu}}$. Then $\left|\frac{1}{t} \zeta_{j}\right|<1, j=1, \ldots, \nu$ (use (2)), and

$$
\left|\left(\frac{\zeta_{1}}{t}\right) \ldots\left(\frac{\zeta_{\nu}}{t}\right)\right|=\left|\xi_{1} \ldots \xi_{\mu}\right| .
$$

Hence, we may assume that

$$
\left|\zeta_{1} \ldots \zeta_{\nu}\right|=\left|\xi_{1} \ldots \xi_{\mu}\right|=C<N .
$$

Moreover, replacing $\varphi_{1}(\lambda)$ with $\varphi_{1}\left(e^{-i \theta_{1}} \lambda\right)$ and $\varphi_{2}(\lambda)$ with $\varphi_{2}\left(e^{-i \theta_{2}} \lambda\right)$, where $\theta_{1}, \theta_{2}$ are chosen such that $e^{i \theta_{1}} \zeta_{1} \ldots e^{i \theta_{1}} \zeta_{\nu}=C$ and $e^{i \theta_{2}} \xi_{1} \ldots e^{i \theta_{2}} \xi_{\mu}=C$, we may assume that $\zeta_{1} \ldots \zeta_{\nu}=\xi_{1} \ldots \xi_{\mu}=C$.

We consider Blaschke products

$$
B_{1}(\lambda):=\prod_{j=1}^{\nu} \frac{\zeta_{j}-\lambda}{1-\bar{\zeta}_{j} \lambda}
$$

and

$$
\widetilde{B}_{1}(\lambda)=\frac{B_{1}(\lambda)-B_{1}(0)}{1-\overline{B_{1}}(0) B_{1}(\lambda)}=e^{i \theta} \prod_{j=1}^{\nu} \frac{\lambda-w_{j}}{1-\bar{w}_{j} \lambda}, \quad \lambda \in \bar{E} .
$$

\footnotetext{
${ }^{1}$ Note that mappings $\varphi_{1}$ and $\varphi_{2}$ are holomorphic in some neighborhood of $\bar{E}$, and the sets $\varphi_{1}^{-1}\left(a_{1}\right)$ and $\varphi_{2}^{-1}\left(b_{1}\right)$ may contain points outside of $E$.

${ }^{2}$ For example, it is enough to change very little the mappings $\varphi_{1}$ and $\varphi_{2}$ by the formula (3) given below.
} 
We choose different $w_{j}^{\prime}, 1 \leq j \leq \nu$, as close to $w_{j}$ as we want such that $0 \in$ $\left\{w_{1}^{\prime}, \ldots, w_{\nu}^{\prime}\right\}$. Define

$$
G_{1}(\lambda)=e^{i \theta} \prod_{j=1}^{\nu} \frac{\lambda-w_{j}^{\prime}}{1-\bar{w}_{j}^{\prime} \lambda} .
$$

Note that $\widetilde{B}_{1}^{-1}(-C)=\left\{\zeta_{1}, \ldots, \zeta_{\nu}\right\}$. We can find $w_{1}^{\prime}, \ldots, w_{\nu}^{\prime}$ such that $G_{1}^{-1}(-C)$ consists of $\nu$ different points $\zeta_{j}^{\prime}, 1 \leq j \leq \nu$, as close to points $\zeta_{j}$ as we want. Let us replace the mapping $\varphi_{1}$ with the mapping

$$
\widetilde{\varphi}_{1}(\lambda):=\left(\varphi(\lambda)-a_{1}\right) \frac{\prod_{j=1}^{\nu} \zeta_{j}\left(\lambda-\zeta_{j}^{\prime}\right)}{\prod_{j=1}^{\nu} \zeta_{j}^{\prime}\left(\lambda-\zeta_{j}\right)}+a_{1} .
$$

Clearly, when $\zeta_{j}^{\prime}, 1 \leq j \leq \nu$, are sufficiently close to $\zeta_{j}, \widetilde{\varphi}_{1}$ maps $E$ into $D_{1}$ (recall that $\varphi_{1}$ maps some neighborhood of $\bar{E}$ into $D_{1}$, hence $\varphi_{1}(E) \Subset D_{1}$ ), and $\widetilde{\varphi}_{1}(0)=\varphi_{1}(0), \widetilde{\varphi}_{1}\left(\zeta_{j}^{\prime}\right)=\varphi_{1}\left(\zeta_{j}\right)$.

Repeating this process for $\varphi_{2}$, we may assume that for Blaschke products $B_{1}$ and $B_{2}$ derivatives are not equal to 0 either on preimages of $C$ or at points $\zeta_{j}$ or $\xi_{j}$ respectively.

Let $A$ be the union of images of singular points under mappings $B_{1}$ and $B_{2}$. Note that neither 0 nor $C$ is in $A$. Let $\pi$ be a holomorphic universal covering of $E \backslash A$ by $E$ with $\pi(0)=C$. There are liftings $\psi_{1}$ and $\psi_{2}$ mapping $E$ into $E$ such that $\pi=B_{1} \circ \psi_{1}=B_{2} \circ \psi_{2}$ and $\psi_{1}(0)=\psi_{2}(0)=0$. If $\pi^{-1}(0)=\left\{\eta_{1}, \eta_{2}, \ldots\right\}$, then mappings $\varphi_{1} \circ \psi_{1}$ and $\varphi_{2} \circ \psi_{2}$ map 0 into $a_{2}$ and $b_{2}$, and all points $\eta_{j}$ into $a_{1}$ and $b_{1}$ respectively.

Note that $\pi$ has all radial limits either in $\partial E$ or in $A$. Since $A$ is finite, $\pi$ is an inner function. By Theorem 2 of Ch. III in [Nos], every inner function which has no zero radial limits is a Blaschke product. Thus

$$
\pi(\lambda)=e^{i \alpha} \prod_{j=1}^{\infty} \frac{\bar{\eta}_{j}}{\left|\eta_{j}\right|} \frac{\eta_{j}-\lambda}{1-\bar{\eta}_{j} \lambda}
$$

and

$$
\left|\prod_{j=1}^{\infty} \eta_{j}\right|=\pi(0)=C<N .
$$

Since $\left(\varphi_{1} \circ \psi_{1}, \varphi_{2} \circ \psi_{2}\right)$ maps $E$ into $D_{1} \times D_{2}$,

$$
g_{D_{1} \times D_{2}}\left(\left(a_{1}, b_{1}\right),\left(a_{2}, b_{2}\right)\right) \leq \prod_{j=1}^{\infty}\left|\eta_{j}\right|<N .
$$

\section{ACKNOWLEDGEMENT}

I am very grateful to Professors M. Jarnicki, P. Pflug, and W. Zwonek for helpful discussions and remarks. I also would like to thank the referee, who turned my attention to the book [Nos] and whose suggestions improved this paper. 


\section{REFERENCES}

[Jar-Pfl1] M. Jarnicki and P. Pflug, Invariant Distances and Metrics in Complex Analysis, Walter de Gruyter, 1993. MR 94k:32039

[Jar-Pfl2] M. Jarnicki and P. Pflug, Remarks on the pluricomplex Green function, Indiana Univ. Math. Journal 44 (2) (1995), 535-543. MR 96k:32026

[Nos] K. Noshiro, Cluster sets, Springer-Verlag, 1960. MR 24:A3295

[Pol] E. Poletsky, Holomorphic currents, Indiana Univ. Math. Journal 42 (1) (1993), 85-144. MR 94c:32007

Instytut Matematyki, Uniwersytet Jagielloński, Reymonta 4, 30-059 Kraków, POLAND

E-mail address: edigaria@im.uj.edu.pl 